\title{
Anisotropic Elastoplastic Damage Mechanics Method to Predict Fatigue Life of the Structure
}

\author{
Hualiang Wan, Qizhi Wang, and Zheng Zhang \\ Institute of Solid Mechanics, Beihang University, Beijing 100191, China \\ Correspondence should be addressed to Qizhi Wang; wangqizhibuaa@163.com
}

Received 31 May 2016; Revised 14 August 2016; Accepted 5 September 2016

Academic Editor: Luciano Lamberti

Copyright (C) 2016 Hualiang Wan et al. This is an open access article distributed under the Creative Commons Attribution License, which permits unrestricted use, distribution, and reproduction in any medium, provided the original work is properly cited.

\begin{abstract}
New damage mechanics method is proposed to predict the low-cycle fatigue life of metallic structures under multiaxial loading. The microstructure mechanical model is proposed to simulate anisotropic elastoplastic damage evolution. As the micromodel depends on few material parameters, the present method is very concise and suitable for engineering application. The material parameters in damage evolution equation are determined by fatigue experimental data of standard specimens. By employing further development on the ANSYS platform, the anisotropic elastoplastic damage mechanics-finite element method is developed. The fatigue crack propagation life of satellite structure is predicted using the present method and the computational results comply with the experimental data very well.
\end{abstract}

\section{Introduction}

For multiaxial fatigue problem, many methods [1-4] have been developed over the past decades. A series of fatigue failure approaches are classified as follows: the linear rule method [5], the equivalent stress method [6], the critical plane method $[7,8]$, the stress invariant method [9], the damage mechanics method [10-16], and so on. However, there is no existing method accepted extensively in the engineering field at present. Hence, further work still must be done. Recently, there is a trend in favor of both the critical plane approach and the damage mechanics approach.

The critical plane method $[17,18]$ can predict the fatigue crack orientation. For the damage mechanics method [15, 19], a great deal of attention has been paid. The damage mechanics method [13, 14, 20-23] deals with the mechanical behavior of the deteriorated materials. Damage models can be classified as follows: isotropic damage and anisotropic damage. As the isotropic damage model is characterized with simple constitutive relationship, it has been widely applied in the engineering field. For isotropic damage model, damage mechanicsfinite element method [19] is proposed to predict the fatigue life of the engineering structure. However, most of the fatigue problem is anisotropic. Thus anisotropic damage model will be more valuable. Many models [24-26] have been proposed to describe anisotropic damage properties of materials. An elastic microstructure model in [27] is proposed to evaluate the anisotropic elastic fatigue problem. Some models [11, 12] for dealing with elastoplastic fatigue problems are developed. However, in engineering applications we do not find extensive applications of them because isotropic schemes can be put more easily into available computer codes, although such approaches are less realistic. Thus, for the low-cycle fatigue problem, it will be very valuable to propose an anisotropic elastoplastic model for the engineering application.

In this paper, damage mechanics method is proposed to predict the low-cycle fatigue life of metallic structures. Referring to the elastic micromodel [27] and the critical plane method [17, 18], a micro elastoplastic mechanical model is established. Considering the hysteresis energy [28], the damage evolution equation is advanced. The material parameters in the damage evolution equation are determined by the low-cycle fatigue experimental data of standard specimens. Based on the further development on the ANSYS platform, anisotropic elastoplastic damage mechanics-finite element method is developed. In this paper, the low-cycle fatigue crack initiation and propagation life of the structure are predicted using the present method. For a real component of 
satellite structure, the fatigue life is predicted by the present method.

\section{The Anisotropic Elastoplastic Damage Theory}

2.1. The Microstructure Mechanical Model. In this section, a microstructure mechanical model is proposed to simulate the anisotropic elastoplastic damage failure. The microstructure mechanical model, as shown in Figure 1, is constituted by elastic block and boom-panel structure unit. Boom-structure is used to simulate the damage failure and elastic block does not undergo damage. For boom-panel structure model, boom is used to transfer the tensile and compressive loading and panel is used to transfer the shear loading.

As we all know, for the metallic materials, the iterative appearance of the shear slip will result in the crack initiation. Boom-panel structure in the micromodel simulates shearing slip band. The present micromodel is built at three orthogonal planes with maximum shear stress. Then the relationship between the micromodel and each element in the structure is built and shown in Figure 2. Global coordinate system belongs to the element in the structure and local coordinate system belongs to the micromodel.

2.2. Stress-Strain Relationships for the Micromodel. The minimum cell of the micromodel is shown in Figure 3. Based on the minimum cell in Figure 3, parameters involved in the micromodel will be discussed in detail in this section.

In the local coordinate system, the points for the micromodel are as follows:

$$
\begin{aligned}
& \varepsilon_{i j}=\left(\frac{l_{1}}{l_{1}+l_{2}}\right) \cdot \varepsilon_{i j 1}+\left(\frac{l_{2}}{l_{1}+l_{2}}\right) \cdot \varepsilon_{i j 2} \\
& \sigma_{i j}=\left(\frac{l_{1}}{l_{1}+l_{2}}\right)^{2} \cdot \sigma_{i j 1}=\left(\frac{l_{2}}{l_{1}+l_{2}}\right)^{2} \cdot \sigma_{i j 2} .
\end{aligned}
$$

$\sigma_{i j}, \varepsilon_{i j}$ denote the stress tensor components and the strain tensor components of the micromodel, respectively, $\sigma_{i j 1}, \varepsilon_{i j 1}$ denote the stress tensor components and the strain tensor components of elastic block, respectively, $\sigma_{i j 2}, \varepsilon_{i j 2}$ denote the stress tensor components and the strain tensor components of boom-panel structure, respectively, $l_{1}$ is the length of elastic block, and $l_{2}$ is the length of boom-panel structure.

2.3. Elastic Analysis of the Micromodel. For elastic block, the linear elastic constitutive model is expressed as

$$
\varepsilon_{i j 1}=\frac{1}{E_{1}}\left(1+\mu_{1}\right) \cdot \sigma_{i j 1}-\delta_{i j} \cdot \frac{\mu_{1}}{E_{1}} \cdot \sigma_{k k 1} .
$$

For boom-panel structure, an elastic analysis is written as

$$
\begin{aligned}
& \varepsilon_{x 2}=\frac{1}{K_{F}} \cdot \sigma_{x 2} \\
& \varepsilon_{y 2}=\frac{1}{K_{F}} \cdot \sigma_{y 2} \\
& \varepsilon_{z 2}=\frac{1}{K_{F}} \cdot \sigma_{z 2}
\end{aligned}
$$

for the boom

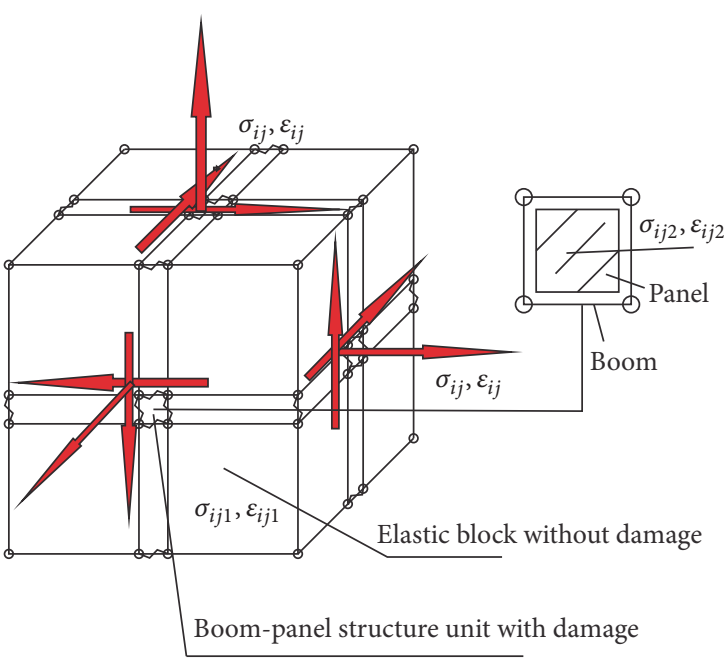

FIgURE 1: The microstructure mechanical model.

$$
\begin{aligned}
& \varepsilon_{x y 2}=\frac{1}{K_{S 1}} \cdot \tau_{x y 2} \\
& \varepsilon_{y z 2}=\frac{1}{K_{S 1}} \cdot \tau_{y z 2} \\
& \varepsilon_{x z 2}=\frac{1}{K_{S 1}} \cdot \tau_{x z 2}
\end{aligned}
$$

for the panel

in which $K_{S 1}$ is the elastic shear modulus of panel and $K_{F}$ is the elastic modulus of boom.

2.3.1. The Analysis of the Elastic Constitutive Relationship. In the local coordinate system, the elastic part of constitutive model of the micromodel is expressed as

$$
\begin{aligned}
\varepsilon_{x}= & \frac{1}{E_{1}} \cdot\left(\frac{l_{1}+l_{2}}{l_{1}}\right) \cdot\left[\sigma_{x}-\mu_{1} \cdot\left(\sigma_{y}+\sigma_{z}\right)\right]+\frac{1}{K_{F}} \\
& \cdot\left(\frac{l_{1}+l_{2}}{l_{2}}\right) \cdot \sigma_{x} \\
\varepsilon_{y}= & \frac{1}{E_{1}} \cdot\left(\frac{l_{1}+l_{2}}{l_{1}}\right) \cdot\left[\sigma_{y}-\mu_{1} \cdot\left(\sigma_{x}+\sigma_{z}\right)\right]+\frac{1}{K_{F}} \\
& \cdot\left(\frac{l_{1}+l_{2}}{l_{2}}\right) \cdot \sigma_{y} \\
\varepsilon_{z}= & \frac{1}{E_{1}} \cdot\left(\frac{l_{1}+l_{2}}{l_{1}}\right) \cdot\left[\sigma_{z}-\mu_{1} \cdot\left(\sigma_{x}+\sigma_{y}\right)\right]+\frac{1}{K_{F}} \\
& \cdot\left(\frac{l_{1}+l_{2}}{l_{2}}\right) \cdot \sigma_{z} \\
\varepsilon_{x y}= & {\left[\frac{1}{2 G_{1}} \cdot\left(\frac{l_{1}+l_{2}}{l_{1}}\right)+\frac{1}{K_{S 1}} \cdot\left(\frac{l_{1}+l_{2}}{l_{2}}\right)\right] \cdot \tau_{x y} } \\
\varepsilon_{x z}= & {\left[\frac{1}{2 G_{1}} \cdot\left(\frac{l_{1}+l_{2}}{l_{1}}\right)+\frac{1}{K_{S 1}} \cdot\left(\frac{l_{1}+l_{2}}{l_{2}}\right)\right] \cdot \tau_{x z} }
\end{aligned}
$$




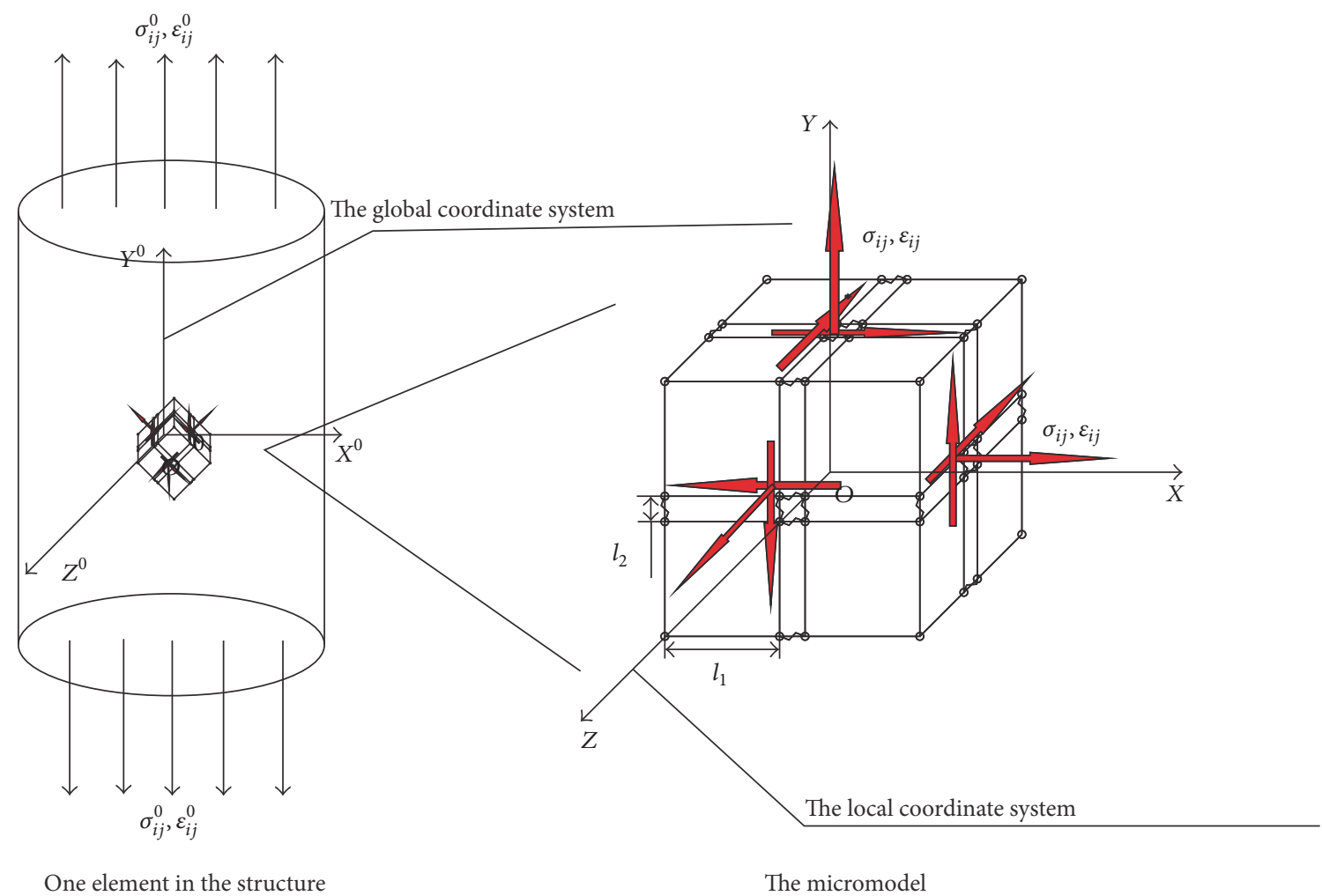

FIGURE 2: The relationship between one element in the structure and the micromodel.

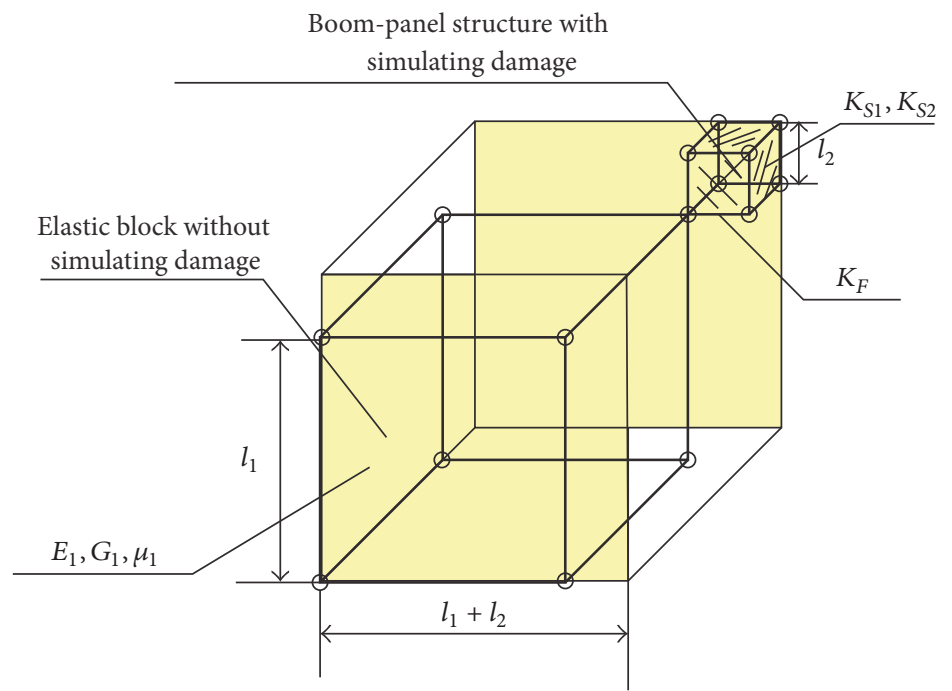

FIGURE 3: The minimum cell of the micromodel.

$$
\varepsilon_{y z}=\left[\frac{1}{2 G_{1}} \cdot\left(\frac{l_{1}+l_{2}}{l_{1}}\right)+\frac{1}{K_{S 1}} \cdot\left(\frac{l_{1}+l_{2}}{l_{2}}\right)\right] \cdot \tau_{y z}
$$

in which $E_{1}$ is Young's modulus of elastic block, $G_{1}$ is the shear modulus of elastic block, and $\mu_{1}$ is Poisson's ratio of elastic block.
In the global coordinate system, the elastic part of constitutive model can be expressed as

$$
\begin{aligned}
& \varepsilon_{x}^{0}=\frac{1}{E} \cdot\left[\sigma_{x}^{0}-\mu \cdot\left(\sigma_{y}^{0}+\sigma_{z}^{0}\right)\right] \\
& \varepsilon_{y}^{0}=\frac{1}{E} \cdot\left[\sigma_{y}^{0}-\mu \cdot\left(\sigma_{x}^{0}+\sigma_{z}^{0}\right)\right]
\end{aligned}
$$




$$
\begin{aligned}
& \varepsilon_{z}^{0}=\frac{1}{E} \cdot\left[\sigma_{z}^{0}-\mu \cdot\left(\sigma_{x}^{0}+\sigma_{y}^{0}\right)\right] \\
& \varepsilon_{x y}^{0}=\frac{1}{2 G} \cdot \tau_{x y}^{0} \\
& \varepsilon_{x z}^{0}=\frac{1}{2 G} \cdot \tau_{x z}^{0} \\
& \varepsilon_{y z}^{0}=\frac{1}{2 G} \cdot \tau_{y z}^{0}
\end{aligned}
$$

in which $\sigma_{i j}^{0}, \varepsilon_{i j}^{0}$ stand for the stress tensor components and the strain tensor components of one element in the structure, respectively, $E$ is Young's modulus, $G$ is the shear modulus, and $\mu$ is Poisson's ratio.

2.3.2. Elastic Material Parameters of the Micromodel. From (4) and (5), we have

$$
\begin{aligned}
\frac{1}{E} & =\frac{1}{E_{1}} \cdot\left(\frac{l_{1}+l_{2}}{l_{1}}\right)+\frac{1}{K_{F}} \cdot\left(\frac{l_{1}+l_{2}}{l_{2}}\right) \\
\mu & =\frac{\mu_{1} /\left(E_{1} \cdot l_{1}\right)}{1 /\left(E_{1} \cdot l_{1}\right)+1 /\left(K_{F} \cdot l_{2}\right)} \\
& =\frac{\mu_{1}}{1+\left(E_{1} / K_{F}\right) \cdot\left(l_{1} / l_{2}\right)} \\
\frac{1}{2 G} & =\frac{1+\mu}{E}=\left[\frac{1}{2 G_{1}} \cdot\left(\frac{l_{1}+l_{2}}{l_{1}}\right)+\frac{1}{K_{S 1}} \cdot\left(\frac{l_{1}+l_{2}}{l_{2}}\right)\right] .
\end{aligned}
$$

The shear modulus $G_{1}$ of elastic block is also written as

$$
\frac{1}{2 \cdot G_{1}}=\frac{1+\mu_{1}}{E_{1}} .
$$

From (6) and (7), then

$$
\begin{aligned}
K_{F} & =E \cdot(1+\mu) \cdot\left(\frac{\mu_{1}}{\mu_{1}-\mu}\right)^{2} \\
E_{1} & =E \cdot \frac{1+\mu}{1+\mu_{1}} \cdot\left(\frac{\mu_{1}}{\mu}\right)^{2} \\
\frac{1}{2 G_{1}} & =\frac{1}{E \cdot(1+\mu)} \cdot\left[\frac{\left(1+\mu_{1}\right) \cdot \mu}{\mu_{1}}\right]^{2} \\
\frac{1}{K_{S 1}} & =\frac{1}{E \cdot(1+\mu)} \cdot\left(\frac{\mu_{1}-\mu}{\mu_{1}}\right)^{2} \\
\frac{l_{1}+l_{2}}{l_{1}} & =1+c=\frac{1+\mu}{1+\mu_{1}} \frac{\mu_{1}}{\mu} \\
\frac{l_{1}+l_{2}}{l_{2}} & =1+\frac{1}{c}=\frac{\mu_{1}}{\mu_{1}-\mu}(1+\mu) \\
\mu_{1} & =\frac{\mu \cdot(1+c)}{1-\mu \cdot c},
\end{aligned}
$$

in which $E, \mu$ are known and $c=l_{2} / l_{1}$ is a constant. Then $K_{F}$, $E_{1}, G_{1}, K_{S 1}, \mu_{1}, l_{1}$, and $l_{2}$ are known.

$c$ constant is a material parameter that can vary in the range defined by (26).

\subsection{Plastic Analysis of the Micromodel}

2.4.1. The Elastoplastic Constitutive Equation. For the micromodel in Figure 3, block is elastic and boom-panel structure is elastoplastic. For boom-panel structure, panel is assumed as bilinear plastic and boom is elastic. For the flow rule of the plastic behavior, the kinematic hardening law is adopted in this article. In the local coordinate system, the elastoplastic constitutive relation of the micromodel is expressed as

$$
\begin{aligned}
\varepsilon_{x}= & \frac{1}{E_{1}} \cdot\left(\frac{l_{1}+l_{2}}{l_{1}}\right) \cdot\left[\sigma_{x}-\mu_{1} \cdot\left(\sigma_{y}+\sigma_{z}\right)\right]+\frac{1}{K_{F}} \\
& \cdot\left(\frac{l_{1}+l_{2}}{l_{2}}\right) \cdot \sigma_{x} \\
\varepsilon_{y}= & \frac{1}{E_{1}} \cdot\left(\frac{l_{1}+l_{2}}{l_{1}}\right) \cdot\left[\sigma_{y}-\mu_{1} \cdot\left(\sigma_{x}+\sigma_{z}\right)\right]+\frac{1}{K_{F}} \\
& \cdot\left(\frac{l_{1}+l_{2}}{l_{2}}\right) \cdot \sigma_{y} \\
\varepsilon_{z}= & \frac{1}{E_{1}} \cdot\left(\frac{l_{1}+l_{2}}{l_{1}}\right) \cdot\left[\sigma_{z}-\mu_{1} \cdot\left(\sigma_{x}+\sigma_{y}\right)\right]+\frac{1}{K_{F}} \\
\varepsilon_{x y}= & \frac{\tau_{x y}}{2 G_{1}} \cdot \frac{l_{1}+l_{2}}{l_{1}}+\left(\varepsilon_{2 S}+\frac{\tau_{x y 2}-\tau_{2 S}}{K_{S 2}}\right) \cdot \frac{l_{2}}{l_{1}+l_{2}} \\
\varepsilon_{x z}= & \frac{\tau_{x z}}{2 G_{1}} \cdot \frac{l_{1}+l_{2}}{l_{1}}+\left(\varepsilon_{2 S}+\frac{\tau_{x z 2}-\tau_{2 S}}{K_{S 2}}\right) \cdot \frac{l_{2}}{l_{1}+l_{2}} \\
\varepsilon_{1} & \frac{l_{1}+l_{2}}{l_{1}}+\left(\varepsilon_{2 S}+\frac{\tau_{y z 2}-\tau_{2 S}}{K_{S 2}}\right) \cdot \frac{l_{2}}{l_{1}+l_{2}},
\end{aligned}
$$

where

$$
\begin{aligned}
\tau_{2 S} & =\frac{\sigma_{s}}{2} \cdot\left(\frac{l_{1}+l_{2}}{l_{2}}\right)^{2} \\
\tau_{x y 2} & =\tau_{x y} \cdot\left(\frac{l_{1}+l_{2}}{l_{2}}\right)^{2} \\
\varepsilon_{2 S} & =\frac{\tau_{2 S}}{K_{S 1}} \cdot\left(\frac{l_{1}+l_{2}}{l_{2}}\right)^{2}
\end{aligned}
$$

in which $\tau_{2 S}$ is the shear yield stress of boom-panel structure, $\varepsilon_{2 S}$ indicates the shear yield strain of boom-panel structure, and $K_{S 2}$ signifies the plastic shear modulus of panel.

2.4.2. Plastic Material Parameters of the Micromodel. In order to determine $K_{S 2}$, a simple stress state is considered. Schematic of a plate subject to tension is shown in 
Figure 4. The structure is in plane-stress condition. For this case, all of the micromodels are the same and built at the position with $\alpha=45^{\circ}$.

In the global coordinate system, the strain $\varepsilon^{0}$ of the structure is defined as

$$
\varepsilon^{0}=\frac{\Delta u_{y}^{0}}{\sqrt{2} \cdot\left(l_{1}+l_{2}\right)}
$$

in which $\Delta u_{y}^{0}$ is the displacement increment of the micromodel along $Y^{0}$ direction in the global coordinate system.

By the coordinate system transformation, the displacement increment $\Delta u_{y}^{0}$ is expressed as

$$
\Delta u_{y}^{0}=\frac{\sqrt{2}}{2} \cdot\left(\Delta u_{x}+\Delta u_{y}\right)
$$

in which

$$
\begin{aligned}
& \Delta u_{x}=\varepsilon_{x} \cdot\left(l_{1}+l_{2}\right)+\varepsilon_{x y} \cdot\left(l_{1}+l_{2}\right) \\
& \Delta u_{y}=\varepsilon_{y} \cdot\left(l_{1}+l_{2}\right)+\varepsilon_{x y} \cdot\left(l_{1}+l_{2}\right)
\end{aligned}
$$

in which $\Delta u_{x}, \Delta u_{y}$ are the displacement increment of the micromodel along $X$ or $Y$ direction in the local coordinate system, respectively.

The stress values $\sigma_{x}, \sigma_{y}$, and $\tau_{x y}$ in the local coordinate system are

$$
\begin{aligned}
& \sigma_{x}=\sigma_{y}=\frac{\sigma^{0}}{2}, \\
& \tau_{x y}=\frac{\sigma^{0}}{2} .
\end{aligned}
$$

From (15)-(20), then the strain $\varepsilon^{0}$ of the structure is expressed as

$$
\begin{aligned}
\varepsilon^{0}= & \frac{\varepsilon_{x}+\varepsilon_{y}}{2}+\varepsilon_{x y} \\
= & {\left[\frac{1}{E}+\frac{l_{1}+l_{2}}{2 \cdot l_{2}} \cdot\left(\frac{1}{K_{S 2}}-\frac{1}{K_{S 1}}\right)\right] \cdot \sigma^{0}-\frac{l_{1}+l_{2}}{2 \cdot l_{2}} } \\
& \cdot\left(\frac{1}{K_{S 2}}-\frac{1}{K_{S 1}}\right) \cdot \sigma_{s} .
\end{aligned}
$$

In the global coordinate system, the strain $\varepsilon^{0}$ of the structure is expressed as

$$
\varepsilon^{0}=\varepsilon_{s}+\frac{\sigma^{0}-\sigma_{s}}{K_{2}}=\frac{\sigma_{s}}{E}+\frac{\sigma^{0}-\sigma_{s}}{K_{2}} .
$$

From (21) and (22), the following requirement must be satisfied:

$$
\frac{\varepsilon_{x}+\varepsilon_{y}}{2}+\varepsilon_{x y}=\varepsilon_{s}+\frac{\sigma^{0}-\sigma_{s}}{K_{2}} .
$$

Then we have

$$
\frac{1}{E}+\frac{l_{1}+l_{2}}{2 \cdot l_{2}} \cdot\left(\frac{1}{K_{S 2}}-\frac{1}{K_{S 1}}\right)=\frac{1}{K_{2}} .
$$

From (11), (13), and (24), then the inverse of the plastic shear modulus $K_{S 2}$ is

$$
\begin{aligned}
\frac{1}{K_{S 2}}= & \left(\frac{1}{K_{2}}-\frac{1}{E}\right) \cdot \frac{2 \cdot\left(\mu_{1}-\mu\right)}{\mu_{1} \cdot(1+\mu)}+\frac{1}{E \cdot(1+\mu)} \\
& \cdot\left(\frac{\mu_{1}-\mu}{\mu_{1}}\right)^{2} .
\end{aligned}
$$

For the plastic shear modulus $K_{S 2}$, the condition $0 \leq K_{S 2}<$ $K_{S 1}$ must be satisfied. From (11) and (25), the range of the constant $c$ is

$$
c \geq \frac{2 \cdot\left(K_{2}-E\right)}{K_{2} \cdot E+2 \cdot E-K_{2}} .
$$

Substituting (8)-(14), (16), and (25) into (15), we obtain

$$
\begin{aligned}
\varepsilon_{x}= & \frac{1}{E} \cdot\left[\sigma_{x}-\mu \cdot\left(\sigma_{y}+\sigma_{z}\right)\right] \\
\varepsilon_{y}= & \frac{1}{E} \cdot\left[\sigma_{y}-\mu \cdot\left(\sigma_{x}+\sigma_{z}\right)\right] \\
\varepsilon_{z}= & \frac{1}{E} \cdot\left[\sigma_{z}-\mu \cdot\left(\sigma_{x}+\sigma_{y}\right)\right] \\
\varepsilon_{x y}= & {\left[\frac{1}{2 G}+2 \cdot\left(\frac{1}{K_{2}}-\frac{1}{E}\right)\right] \cdot \tau_{x y}-2 \cdot\left(\frac{1}{K_{2}}-\frac{1}{E}\right) } \\
& \cdot \tau_{S} \\
\varepsilon_{y z}= & {\left[\frac{1}{2 G}+2 \cdot\left(\frac{1}{K_{2}}-\frac{1}{E}\right)\right] \cdot \tau_{y z}-2 \cdot\left(\frac{1}{K_{2}}-\frac{1}{E}\right) } \\
& \cdot \tau_{S} \\
\varepsilon_{x z}= & {\left[\frac{1}{2 G}+2 \cdot\left(\frac{1}{K_{2}}-\frac{1}{E}\right)\right] \cdot \tau_{x z}-2 \cdot\left(\frac{1}{K_{2}}-\frac{1}{E}\right) } \\
& \cdot \tau_{S}
\end{aligned}
$$

in which $\tau_{S}$ is a variable and $\tau_{S}=\sigma_{S} / 2$.

2.5. Damage Constitutive Law for the Micromodel. For this micromodel, elastic block does not undergo damage and boom-panel structure can simulate the damage failure. In this section, a simple case in Figure 4 is discussed first. For this case, the micromodel is at the position with $\alpha=45^{\circ}$ or $135^{\circ}$. For boom-panel structure, the constitutive law coupling with damage is displayed in Figure 5.

For a plate subject to uniaxial loading, constitutive equations including damage can be written as follows:

(i) When $\tau_{x y 2} \leq \tau_{2 S} \cdot\left(1-D_{x y}\right)$,

$$
\begin{aligned}
\varepsilon_{x D}= & \frac{1}{E_{1}} \cdot\left(\frac{l_{1}+l_{2}}{l_{1}}\right) \cdot\left[\sigma_{x}-\mu_{1} \cdot \sigma_{y}\right] \\
& +\frac{1}{K_{F} \cdot\left(1-D_{x}\right)} \cdot\left(\frac{l_{1}+l_{2}}{l_{2}}\right) \cdot \sigma_{x} .
\end{aligned}
$$




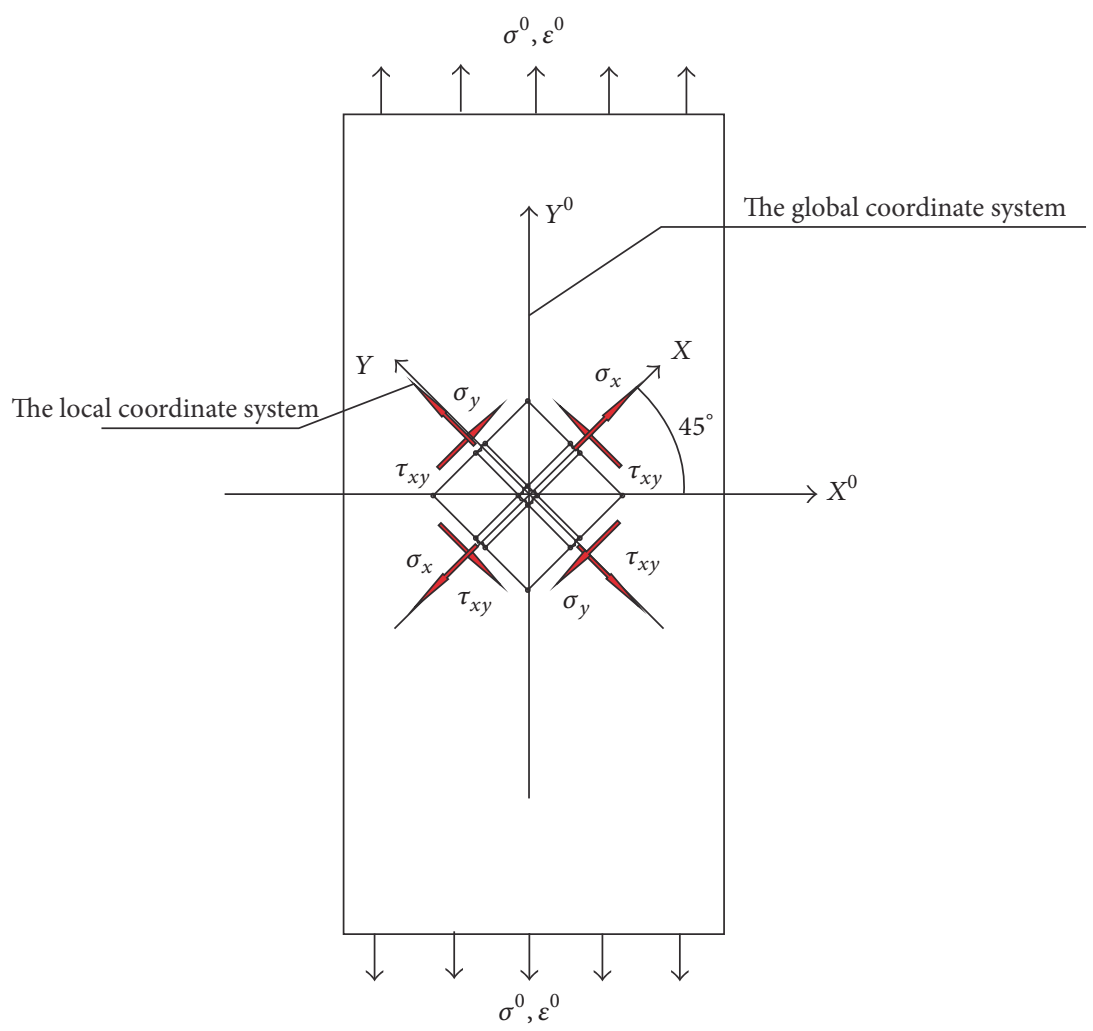

FIGURE 4: Schematic of a plate subject to tension.
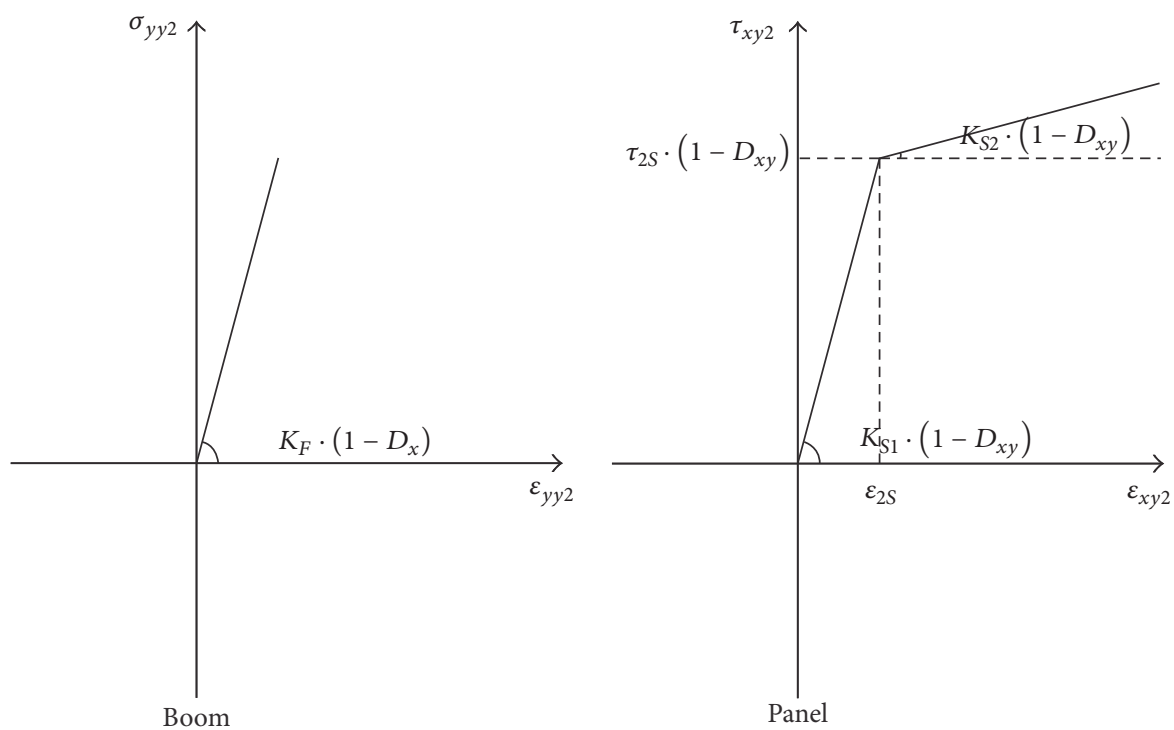

FIGURE 5: The constitutive law with damage for boom-panel structure.

$$
\begin{aligned}
\varepsilon_{y D}= & \frac{1}{E_{1}} \cdot\left(\frac{l_{1}+l_{2}}{l_{1}}\right) \cdot\left[\sigma_{y}-\mu_{1} \cdot \sigma_{x}\right] \\
& +\frac{1}{K_{F} \cdot\left(1-D_{y}\right)} \cdot\left(\frac{l_{1}+l_{2}}{l_{2}}\right) \cdot \sigma_{y} \\
\varepsilon_{x y D}= & \frac{\tau_{x y}}{2 G_{1}} \cdot \frac{l_{1}+l_{2}}{l_{1}}+\frac{\tau_{x y}}{K_{S 1} \cdot\left(1-D_{x y}\right)} \cdot \frac{l_{1}+l_{2}}{l_{2}} \\
= & \frac{\tau_{x y}}{2 \cdot G_{1 D}} .
\end{aligned}
$$

(ii) When $\tau_{x y 2}>\tau_{2 S} \cdot\left(1-D_{x y}\right)$,

$$
\begin{aligned}
\varepsilon_{x D}= & \frac{1}{E_{1}} \cdot\left(\frac{l_{1}+l_{2}}{l_{1}}\right) \cdot\left[\sigma_{x}-\mu_{1} \cdot \sigma_{y}\right] \\
& +\frac{1}{K_{F} \cdot\left(1-D_{x}\right)} \cdot\left(\frac{l_{1}+l_{2}}{l_{2}}\right) \cdot \sigma_{x} \\
\varepsilon_{y D}= & \frac{1}{E_{1}} \cdot\left(\frac{l_{1}+l_{2}}{l_{1}}\right) \cdot\left[\sigma_{y}-\mu_{1} \cdot \sigma_{x}\right] \\
& +\frac{1}{K_{F} \cdot\left(1-D_{y}\right)} \cdot\left(\frac{l_{1}+l_{2}}{l_{2}}\right) \cdot \sigma_{y}
\end{aligned}
$$




$$
\begin{aligned}
\varepsilon_{x y D}= & \frac{\tau_{x y}}{2 G_{1}} \cdot \frac{l_{1}+l_{2}}{l_{1}} \\
& +\left(\varepsilon_{2 S}+\frac{\tau_{x y 2}-\tau_{2 S} \cdot\left(1-D_{x y}\right)}{K_{S 2} \cdot\left(1-D_{x y}\right)}\right) \cdot \frac{l_{2}}{l_{1}+l_{2}}
\end{aligned}
$$

in which $D_{x}, D_{y}$ denote the damage variables of boom and $D_{x y}$ denotes the damage variable of panel.

If the plate is subject to multiaxial loading, constitutive equations including damage become

$$
\begin{aligned}
\varepsilon_{x}= & \frac{1}{E_{1}} \cdot\left(\frac{l_{1}+l_{2}}{l_{1}}\right) \cdot\left[\sigma_{x}-\mu_{1} \cdot\left(\sigma_{y}+\sigma_{z}\right)\right] \\
& +\frac{1}{K_{F} \cdot\left(1-D_{x}\right)} \cdot\left(\frac{l_{1}+l_{2}}{l_{2}}\right) \cdot \sigma_{x} \\
\varepsilon_{y}= & \frac{1}{E_{1}} \cdot\left(\frac{l_{1}+l_{2}}{l_{1}}\right) \cdot\left[\sigma_{y}-\mu_{1} \cdot\left(\sigma_{x}+\sigma_{z}\right)\right] \\
& +\frac{1}{K_{F} \cdot\left(1-D_{y}\right)} \cdot\left(\frac{l_{1}+l_{2}}{l_{2}}\right) \cdot \sigma_{y} \\
\mathcal{E}_{z}= & \frac{1}{E_{1}} \cdot\left(\frac{l_{1}+l_{2}}{l_{1}}\right) \cdot\left[\sigma_{z}-\mu_{1} \cdot\left(\sigma_{x}+\sigma_{y}\right)\right] \\
& +\frac{1}{K_{F} \cdot\left(1-D_{z}\right)} \cdot\left(\frac{l_{1}+l_{2}}{l_{2}}\right) \cdot \sigma_{z} \\
\varepsilon_{y z}= & \frac{\tau_{y z}}{2 G_{1}} \cdot \frac{l_{1}+l_{2}}{l_{1}}+\left(\varepsilon_{2 S}+\frac{\tau_{y z 2}-\tau_{S} \cdot\left(1-D_{y z}\right)}{l_{1}+l_{2}} \cdot\left(1-D_{y z}\right)\right. \\
\varepsilon_{x z}= & \frac{\tau_{x y}}{2 G_{1}} \cdot \frac{l_{1}+l_{2}}{l_{1}}+\left(\varepsilon_{2 S}+\frac{\tau_{x y 2}-\tau_{S} \cdot\left(1-D_{x y}\right)}{l_{1}}\right) \\
& \left.\cdot \frac{l_{2}}{l_{1}+l_{2}}\right) \\
& +\left(1-D_{x y}\right)
\end{aligned}
$$

in which $D_{x}, D_{y}, D_{z}$ denote the damage variables of boom and $D_{x y}, D_{x z}, D_{y z}$ denote the damage variables of panel.

2.6. Relationship between Boom Damage and Panel Damage. For a plate subject to uniaxial loading (see Figure 4), the following requirements need to be satisfied:

$$
\begin{aligned}
D_{z} & =0 \\
D_{x z} & =D_{y z}=0 \\
D_{x} & =D_{y} .
\end{aligned}
$$

For the case in Figure 4, the isotropic property is still satisfied in $X Y$ plane when $\tau_{x y 2} \leq \tau_{2 S} \cdot\left(1-D_{x y}\right)$. So the requirement is as follows:

$$
\frac{1+\mu_{12 D}}{E_{1 D}}=\frac{1}{2 \cdot G_{1 D}} .
$$

From (28), we have

$$
\frac{1}{2 \cdot G_{1 D}}=\frac{1}{2 G_{1}} \cdot \frac{l_{1}+l_{2}}{l_{1}}+\frac{1}{K_{S 1} \cdot\left(1-D_{x y}\right)} \cdot \frac{l_{1}+l_{2}}{l_{2}} .
$$

Substituting (10)-(13), (A.1), (32), and (34) into (33), then

$$
\begin{aligned}
\frac{1}{E} & \cdot\left[\mu+\left(\frac{\mu}{\mu_{1}}+\frac{1}{1-D_{x}} \cdot\left(1-\frac{\mu}{\mu_{1}}\right)\right)\right] \\
& =\frac{1}{E} \cdot\left[\frac{\mu \cdot\left(1+\mu_{1}\right)}{\mu_{1}}+\frac{1}{1-D_{x y}} \cdot \frac{\mu_{1}-\mu}{\mu_{1}}\right] .
\end{aligned}
$$

From (32) and (35), we have

$$
D_{x y}=D_{x}=D_{y} .
$$

Similarly, For the plate in the $X Z$ plane, while $D_{x}=0$ and $D_{x y}=D_{x z}=0$

$$
D_{x}=D_{z}=D_{x z}
$$

For the plate in the $Y Z$ plane, while $D_{x}=0$ and $D_{x y}=D_{x z}=$ 0

$$
D_{y}=D_{z}=D_{y z}
$$

In order to satisfy (36)-(38), then the following conclusions are obtained:

$$
\begin{aligned}
& D_{x}=D_{x y}+D_{x z} \\
& D_{y}=D_{x y}+D_{y z} \\
& D_{z}=D_{x z}+D_{y z} .
\end{aligned}
$$

Thus three damage variables $D_{x y}, D_{y z}, D_{x z}$ are independent.

2.7. Damage Evolution Equation. Based on hysteresis energy, the damage evolution model depends on three variables, $D_{x y}$, $D_{x z}, D_{y z}$. Let us now consider the damage equation of the first variable $D_{x y}$. For the micromodel, the damage failure of the materials is simulated by boom-panel structure. The hysteresis loop is considered by the shear stress of panel $\tau_{x y 2}$. The hysteresis loop for the panel of the micromodel is shown in Figure 6. For this method, the same behavior of the hysteresis loop remains in time when fatigue increases.

For each loop, the hysteresis energy of panel is

$$
\begin{aligned}
W_{0}= & 4 \cdot\left(\tau_{x y}-\frac{\sigma_{s}}{2} \cdot\left(1-D_{x y}\right)\right) \cdot\left(\frac{1}{K_{S 2}}-\frac{1}{K_{S 1}}\right) \cdot \frac{\sigma_{s}}{2} \\
& \cdot\left(\frac{l_{1}+l_{2}}{l_{2}}\right)^{4} \cdot
\end{aligned}
$$




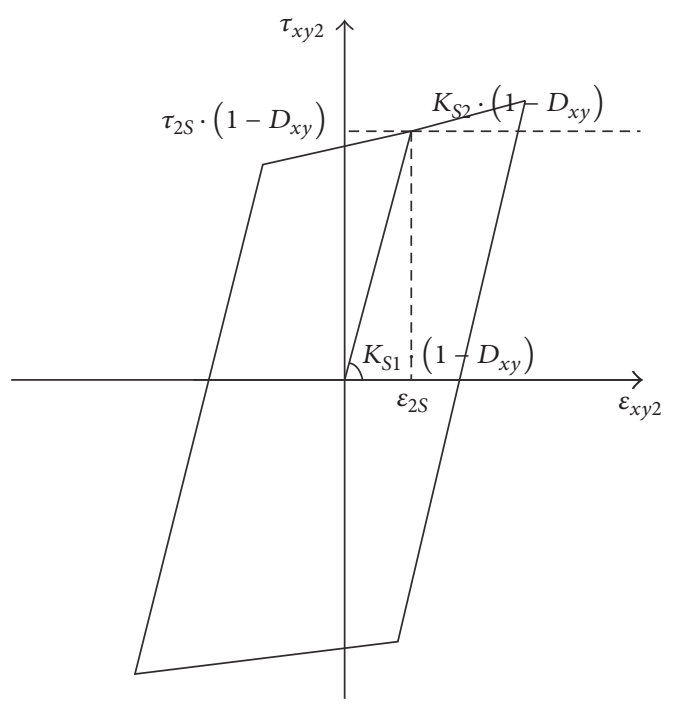

FIGURE 6: The hysteresis loop for the panel in XY plane in the local coordinate system.

The total hysteresis energy of panel is assumed as

$$
W_{c r}=\omega_{c r} \cdot\left(\frac{1}{K_{S 2}}-\frac{1}{K_{S 1}}\right) \cdot \frac{\sigma_{s}}{2} \cdot\left(\frac{l_{1}+l_{2}}{l_{2}}\right)^{4} .
$$

Then the damage evolution equation is defined as

$$
\begin{aligned}
\frac{d D_{x y}}{d N} & =\alpha \cdot \frac{\left(W_{0} / W_{c r}\right)^{m}}{\left(1-D_{x y}\right)^{\beta}} \\
& =\alpha \frac{\left(\tau_{x y}-\left(\sigma_{s} / 2\right) \cdot\left(1-D_{x y}\right)\right)^{m}}{\omega_{c r}^{m} \cdot\left(1-D_{x y}\right)^{\beta}} \\
& =\frac{\left(\tau_{x y}-\left(\sigma_{s} / 2\right) \cdot\left(1-D_{x y}\right)\right)^{m}}{\omega_{c r}^{*} \cdot\left(1-D_{x y}\right)^{\beta}}, \quad\left(\omega_{c r}^{*}=\frac{\alpha}{\omega_{c r}^{m}}\right)
\end{aligned}
$$

in which $\alpha, \beta, m$, and $\omega_{c r}$ are material parameters.

Similarly, the damage evolution equation for the damage variables $D_{x z}, D_{y z}$ can also be obtained. Then the damage evolution equations of the micromodel for three damage variables $D_{x y}, D_{x z}, D_{y z}$ are

$$
\begin{aligned}
\frac{d D_{i j}}{d N} & =\alpha \cdot \frac{\left(W_{0} / W_{c r}\right)^{m}}{\left(1-D_{i j}\right)^{\beta}} \\
& =\alpha \frac{\left(\tau_{i j}-\left(\sigma_{s} / 2\right) \cdot\left(1-D_{i j}\right)\right)^{m}}{\omega_{c r}^{m} \cdot\left(1-D_{i j}\right)^{\beta}} \\
& =\frac{\left(\tau_{i j}-\left(\sigma_{s} / 2\right) \cdot\left(1-D_{i j}\right)\right)^{m}}{\omega_{c r}^{*} \cdot\left(1-D_{i j}\right)^{\beta}}
\end{aligned}
$$

\section{The Damage Mechanics-Finite Element Method}

The present damage mechanics model is implemented in the commercial finite element software ANSYS. Computations proceed as follows:

(1) Stress distribution of the structure is computed first in order to find the critical element.

(2) The increment of damage extent $\Delta D$ of critical element is given, $\Delta D=$ constant, and the magnitude of damage extent increment will be checked by the convergence verification. Then the corresponding fatigue life increments $\Delta N_{x y}(e), \Delta N_{y z}(e)$, and $\Delta N_{x z}(e)$ of critical element are

$$
\begin{aligned}
& \Delta N_{x y}(e)=\frac{\omega_{c r}^{*} \cdot\left(1-D_{x y}\right)^{\beta}}{\left[\tau_{x y}-\left(\sigma_{s} / 2\right) \cdot\left(1-D_{x y}\right)\right]^{m}} \cdot \Delta D \\
& \Delta N_{y z}(e)=\frac{\omega_{c r}^{*} \cdot\left(1-D_{y z}\right)^{\beta}}{\left[\tau_{y z}-\left(\sigma_{s} / 2\right) \cdot\left(1-D_{y z}\right)\right]^{m}} \cdot \Delta D \\
& \Delta N_{x z}(e)=\frac{\omega_{c r}^{*} \cdot\left(1-D_{x z}\right)^{\beta}}{\left[\tau_{x z}-\left(\sigma_{s} / 2\right) \cdot\left(1-D_{x z}\right)\right]^{m}} \cdot \Delta D,
\end{aligned}
$$

where the symbol in right parentheses $(e)$ means critical element and $(x)$ denotes other elements. Subscript without parentheses $x y$ means $X Y$ plane in the local coordinate system.

(3) Then the minimum value $\Delta N_{\min }(e)$ is found and defined as

$\Delta N_{\min }(e)=\min \left\{\Delta N_{x y}(e), \Delta N_{y z}(e), \Delta N_{x z}(e)\right\}$.

(4) From the damage evolution equation (43), the damage variable increment of the elements can be obtained when $\Delta N_{\min }(e)$ is known. The damage extent increments of critical element are

$$
\begin{aligned}
& \Delta D_{x y}(e)=\frac{\left[\tau_{x y}-\left(\sigma_{s} / 2\right) \cdot\left(1-D_{i}\right)\right]^{m}}{\omega_{c r}^{*} \cdot(1-D)^{\beta}} \cdot \Delta N_{\text {min }}(e) . \\
& \Delta D_{y z}(e)=\frac{\left[\tau_{y z}-\left(\sigma_{s} / 2\right) \cdot\left(1-D_{i}\right)\right]^{m}}{\omega_{c r}^{*} \cdot(1-D)^{\beta}} \cdot \Delta N_{\min }(e) \\
& \Delta D_{x z}(e)=\frac{\left[\tau_{x z}-\left(\sigma_{s} / 2\right) \cdot\left(1-D_{i}\right)\right]^{m}}{\omega_{c r}^{*} \cdot(1-D)^{\beta}} \cdot \Delta N_{\min }(e) .
\end{aligned}
$$

Then damage extent increments of other elements are

$$
\begin{aligned}
& \Delta D_{x y}(x)=\frac{\left[\tau_{x y}-\left(\sigma_{s} / 2\right) \cdot\left(1-D_{i}\right)\right]^{m}}{\omega_{c r}^{*} \cdot(1-D)^{\beta}} \cdot \Delta N_{\min }(e) \\
& \Delta D_{y z}(x)=\frac{\left[\tau_{y z}-\left(\sigma_{s} / 2\right) \cdot\left(1-D_{i}\right)\right]^{m}}{\omega_{c r}^{*} \cdot(1-D)^{\beta}} \cdot \Delta N_{\min }(e) \\
& \Delta D_{x z}(x)=\frac{\left[\tau_{x z}-\left(\sigma_{s} / 2\right) \cdot\left(1-D_{i}\right)\right]^{m}}{\omega_{c r}^{*} \cdot(1-D)^{\beta}} \cdot \Delta N_{\min }(e) .
\end{aligned}
$$


(5) Modify the local coordinate system and material properties of damaged elements according to (A.1). The new stress field acting in the structure is determined via FE analysis. At the same time, the level of damage is computed for each element:

$$
\begin{aligned}
& D_{i j}\left(e_{k+1}\right)=D_{i j}\left(e_{k}\right)+\Delta D_{i j}\left(e_{k}\right), \quad(i j=x y, y z, x z) \\
& D_{i j}\left(x_{k+1}\right)=D_{i j}\left(x_{k}\right)+\Delta D_{i j}\left(x_{k}\right), \\
& \\
& \quad(i j=x y, y z, x z)
\end{aligned}
$$

in which $D_{i j}(e)$ is the damage extent of critical element and $D_{i j}(x)$ indicates the damage extent of other elements.

(6) From (39), the damage variables $D_{x}, D_{y}, D_{z}$ of boom can be obtained when $D_{x y}, D_{y z}, D_{x z}$ are known. The process from steps (2) to (5) will be repeated until one of the boom damage extents $D_{x}, D_{y}, D_{z}$ of critical element is equal to 1 . In this step, the failure of critical element means crack initiation in the structure.

(7) Modify local coordinates and material properties of the damaged element and recompute stress distribution by performing a new FE analysis. Determine the next critical element.

(8) Repeat the process from (2) to (7), until the crack length of the engineering structure is equal to $a$. The corresponding fatigue life $N_{a}$ with crack length $a$ is

$$
N_{a}=\sum \Delta N_{\min }(e) \text {. }
$$

\section{Prediction of Fatigue Life}

4.1. Smooth Specimen Made of 35Cr2Ni4MoA. The material parameters in damage evolution equation are determined with low-cycle fatigue experimental data of standard specimens in [29]. The low-cycle fatigue curve $\Delta \varepsilon / 2-2 N f$ of the material $35 \mathrm{Cr} 2 \mathrm{Ni} 4 \mathrm{MoA}$ with stress ratio $R=-1$ is considered in this article. The material parameters of $35 \mathrm{Cr} 2 \mathrm{Ni} 4 \mathrm{MoA}$ are expressed as follows: $E=206.5 \mathrm{GPa}, \mu=0.377$, and $G=$ $75 \mathrm{GPa}$. The material parameters in damage evolution equation are listed in Table 1. Numerical results and experimental data are compared in Figure 7. The results derived from the present model agree with the experimental observations very well.

4.2. Notched Structure Made of $35 \mathrm{Cr} 2 \mathrm{Ni4MoA}$. In this section, a notched structure of $35 \mathrm{Cr} 2 \mathrm{Ni} 4 \mathrm{MoA}$ in Figure 8 is discussed while $K_{T}>1$. The low-cycle fatigue life of the crack initiation and propagation is predicted using the present damage mechanics method. The mean fatigue crack life for the structure of $35 \mathrm{Cr} 2 \mathrm{Ni} 4 \mathrm{MoA}$ is presented in Figure 9. The crack propagation life curve for notched structure is shown in Figure 10 with constant strain $\Delta \varepsilon / 2=2.8 E-3$.

4.3. Real Satellite Structure. In this section, a real satellite structure of 5A06 in Figure 11 is investigated while $R=-1$.

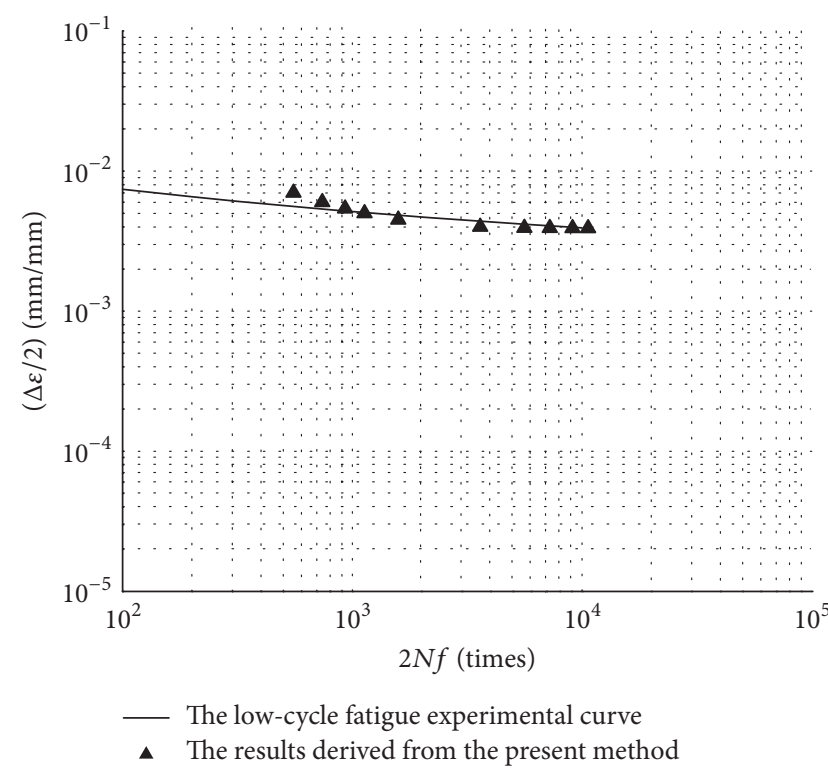

Figure 7: Comparison between FE results and experimental data for material 35Cr2Ni4MoA.

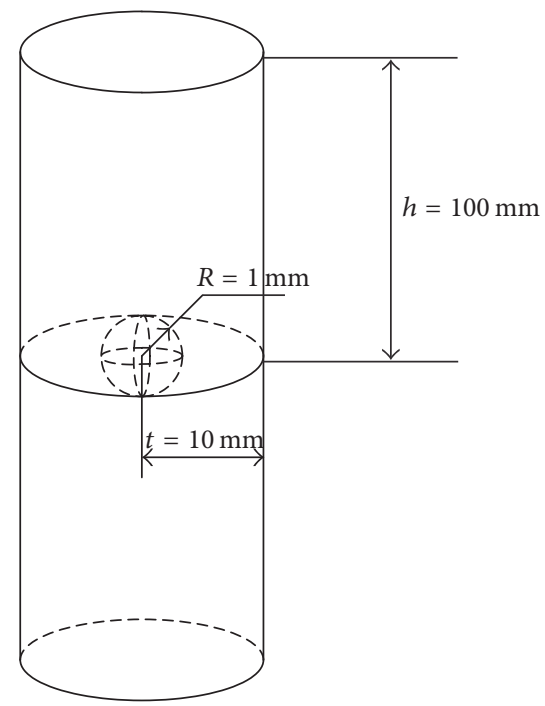

Figure 8: Notched structure of 35Cr2Ni4MoA.

The material parameters in damage evolution equation are listed in Table 2.

The finite element model of real satellite structure is shown in Figure 11. In this paper, the fatigue crack propagation life of real satellite structure is predicted using the present method and shown in Figure 12.

For the satellite structure, the experimental low-cycle fatigue lifetime is as follows: $\log N m=2.7604$ when the crack length $a=4.875 \mathrm{~mm}$.

For the satellite structure, the computational low-cycle fatigue lifetime is as follows: $\log N m=2.7419$ when the crack length $a=4.875 \mathrm{~mm}$.

The relative error between the calculated results and the experimental data is $0.67 \%$. Hence, the fatigue life prediction 


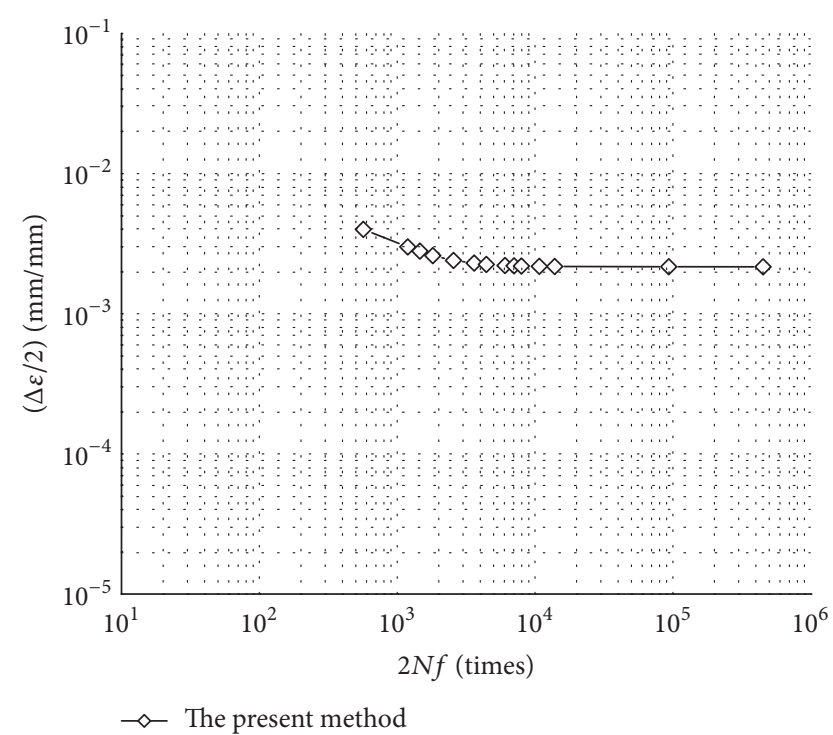

Figure 9: The mean fatigue crack initiation life curve for notched structure.

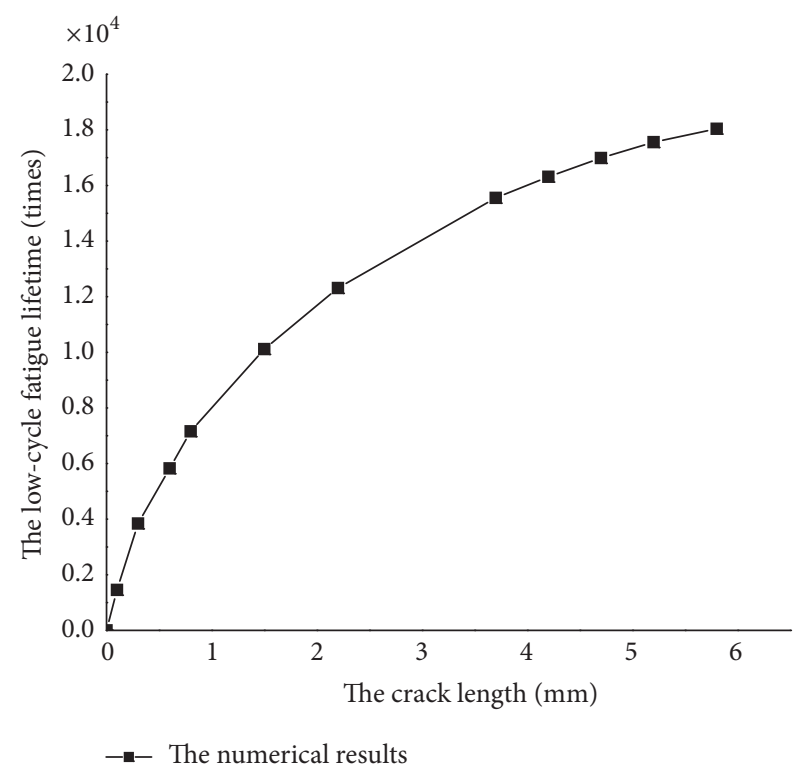

FIGURE 10: The crack propagation curve for notched structure.

TABLE 1: Material properties in damage evolution equation for material 35Cr2Ni4MoA with $K_{T}=1$.

\begin{tabular}{lcc}
\hline$m$ & $\omega_{c r}^{*} /(\mathrm{Pa})^{m}$ & $\beta$ \\
\hline 1.05 & $4.10848 E 11$ & 1.05 \\
\hline
\end{tabular}

for satellite structure is acceptable in the engineering application.
TABLE 2: Material properties in damage evolution equation for material $5 \mathrm{~A} 06$ with $K_{T}=1$.

\begin{tabular}{llc}
\hline$m$ & $\omega_{c r}^{*} /(\mathrm{Pa})^{m}$ & $\beta$ \\
\hline 1.0 & $2.4051 E 10$ & 1.0 \\
\hline
\end{tabular}

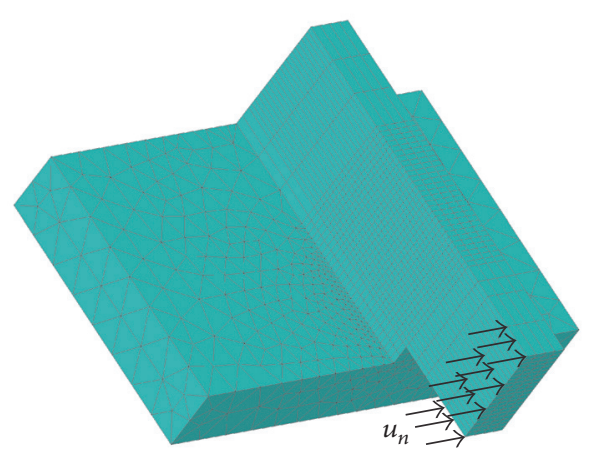

FIGURE 11: The finite element model of satellite structure.

\section{Conclusions}

In this article, a new damage mechanics method is proposed to predict the low-cycle fatigue life of metallic structures under multiaxial loading:

(1) A microstructure mechanical model is proposed to simulate the anisotropic damage failure. As the micromodel depends on few material parameters, the present method is very concise and suitable for engineering application.

(2) Considering the hysteresis energy, the damage evolution equation is constructed. The material parameters are obtained by the low-cycle fatigue experimental results of standard specimens.

(3) Based on the further development on the ANSYS platform, anisotropic elastoplastic damage mechanicsfinite element method is developed.

(4) The fatigue crack initiation and propagation life for notched structure of $35 \mathrm{Cr} 2 \mathrm{Ni} 4 \mathrm{MoA}$ are predicted using the present method.

(5) The fatigue crack growth life of a satellite structure is predicted and the computational results fit well with the experimental data.

\section{Appendix}

Substituting (8)-(14), (16), and (25) into (30), then the elastoplastic constitutive equations including damage are

$$
\begin{aligned}
& \varepsilon_{x}=\frac{1}{E_{1 D}} \cdot \sigma_{x}-\frac{\mu_{12 D}}{E_{2 D}} \cdot \sigma_{y}-\frac{\mu_{13 D}}{E_{3 D}} \cdot \sigma_{z} \\
& \varepsilon_{y}=\frac{1}{E_{2 D}} \cdot \sigma_{y}-\frac{\mu_{21 D}}{E_{1 D}} \cdot \sigma_{x}-\frac{\mu_{23 D}}{E_{3 D}} \cdot \sigma_{z}
\end{aligned}
$$




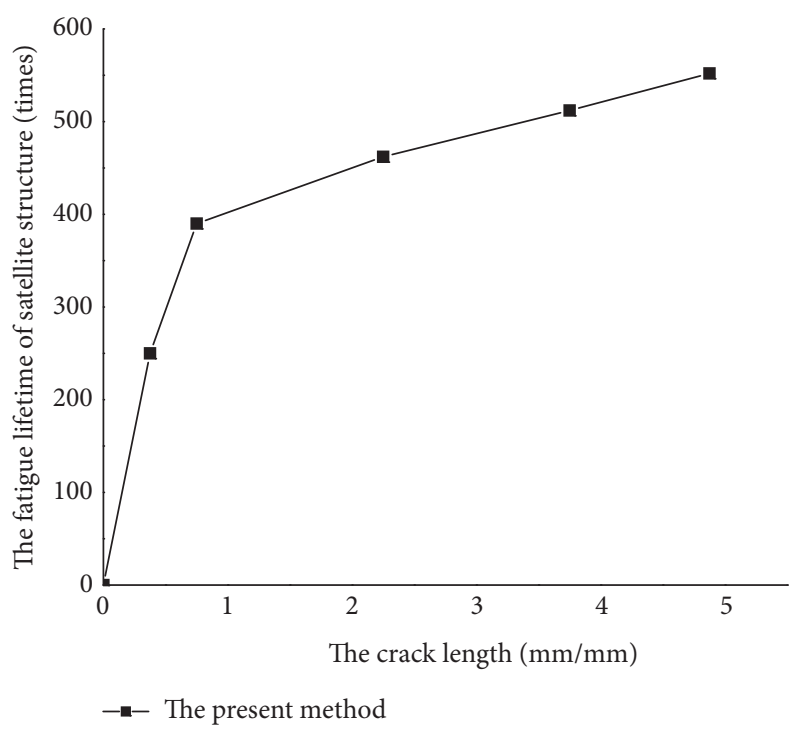

FIGURE 12: The crack propagation curve of satellite structure.

$$
\begin{aligned}
\varepsilon_{z} & =\frac{1}{E_{3 D}} \cdot \sigma_{z}-\frac{\mu_{31 D}}{E_{1 D}} \cdot \sigma_{x}-\frac{\mu_{32 D}}{E_{2 D}} \cdot \sigma_{y} \\
\varepsilon_{x y} & =\left[\frac{1}{2 G} \cdot\left(\frac{\mu}{\mu_{1}} \cdot \frac{1+\mu_{1}}{1+\mu}+\frac{1}{1-D_{x y}} \cdot \frac{\mu_{1}-\mu}{\mu_{1} \cdot(1+\mu)}\right)\right. \\
& \left.+2 \cdot\left(\frac{1}{K_{2}}-\frac{1}{E}\right) \cdot \frac{1}{1-D_{x y}}\right] \cdot \tau_{x y}-2 \cdot\left(\frac{1}{K_{2}}\right. \\
& \left.-\frac{1}{E}\right) \cdot \tau_{S}
\end{aligned}
$$$$
\varepsilon_{y z}=\left[\frac{1}{2 G} \cdot\left(\frac{\mu}{\mu_{1}} \cdot \frac{1+\mu_{1}}{1+\mu}+\frac{1}{1-D_{x y}} \cdot \frac{\mu_{1}-\mu}{\mu_{1} \cdot(1+\mu)}\right)\right.
$$$$
\left.+2 \cdot\left(\frac{1}{K_{2}}-\frac{1}{E}\right) \cdot \frac{1}{1-D_{x y}}\right] \cdot \tau_{y z}-2 \cdot\left(\frac{1}{K_{2}}\right.
$$$$
\left.-\frac{1}{E}\right) \cdot \tau_{S}
$$$$
\varepsilon_{x z}=\left[\frac{1}{2 G} \cdot\left(\frac{\mu}{\mu_{1}} \cdot \frac{1+\mu_{1}}{1+\mu}+\frac{1}{1-D_{x y}} \cdot \frac{\mu_{1}-\mu}{\mu_{1} \cdot(1+\mu)}\right)\right.
$$$$
\left.+2 \cdot\left(\frac{1}{K_{2}}-\frac{1}{E}\right) \cdot \frac{1}{1-D_{x y}}\right] \cdot \tau_{x z}-2 \cdot\left(\frac{1}{K_{2}}\right.
$$$$
\left.-\frac{1}{E}\right) \cdot \tau_{S}
$$

where

$$
\begin{aligned}
& \frac{1}{E_{1 D}}=\frac{1}{E} \cdot\left[\frac{\mu}{\mu_{1}}+\left(1-\frac{\mu}{\mu_{1}}\right) \cdot \frac{1}{1-D_{x}}\right] \\
& \frac{1}{E_{2 D}}=\frac{1}{E} \cdot\left[\frac{\mu}{\mu_{1}}+\left(1-\frac{\mu}{\mu_{1}}\right) \cdot \frac{1}{1-D_{y}}\right]
\end{aligned}
$$

$$
\begin{aligned}
& \frac{1}{E_{3 D}}=\frac{1}{E} \cdot\left[\frac{\mu}{\mu_{1}}+\left(1-\frac{\mu}{\mu_{1}}\right) \cdot \frac{1}{1-D_{z}}\right] \\
& \mu_{21 D}=\mu_{31 D}=\frac{\mu}{\mu / \mu_{1}+\left(1-\mu / \mu_{1}\right) \cdot\left(1 /\left(1-D_{x}\right)\right)} \\
& \mu_{12 D}=\mu_{32 D}=\frac{\mu}{\mu / \mu_{1}+\left(1-\mu / \mu_{1}\right) \cdot\left(1 /\left(1-D_{y}\right)\right)} \\
& \mu_{13 D}=\mu_{23 D}=\frac{\mu}{\mu / \mu_{1}+\left(1-\mu / \mu_{1}\right) \cdot\left(1 /\left(1-D_{z}\right)\right)} .
\end{aligned}
$$

\section{Nomenclature}

$l_{1}:$

$l_{2}:$

a:

$K_{F}$ :

$E_{1}$ :

$G_{1}$ :

$\mu_{1}$ :

$K_{S 1}:$

$K_{S 2}$ :

$\varepsilon_{2 S}$ :

$\sigma_{i j}, \varepsilon_{i j}$ :

$\sigma_{i j 1}, \varepsilon_{i j 1}:$

$\sigma_{i j 2}, \varepsilon_{i j 2}:$

$\sigma_{i j}^{0}, \varepsilon_{i j}^{0}$

$D_{x}, D_{y}, D_{z}$ :

$D_{x y}, D_{y z}, D_{x z}$ :

E:

$K_{2}$ :

$G$ :

$\mu:$
The geometrical length of elastic block

The length of the boom-panel structure

The crack length

The elastic modulus of boom

Young's modulus of elastic block

The shear modulus of elastic block

Poisson's ratio of elastic block

The elastic shear modulus of panel

The plastic shear modulus of panel

The yield strain of panel

The stress tensor components and the strain tensor components of the micromodel in the local coordinate system, respectively The stress tensor components and the strain tensor components of elastic block in the local coordinate system, respectively The stress tensor components and the strain tensor components of boom-panel structure in the local coordinate system, respectively

The stress tensor components and the strain tensor components in the global coordinate system, respectively

The damage variables of boom

The damage variables of panel

Young's modulus

The plastic modulus

The shear modulus

Poisson's ratio.

\section{Competing Interests}

The authors declare that they have no competing interests.

\section{References}

[1] A. Cristofori, L. Susmel, and R. Tovo, "A stress invariant based criterion to estimate fatigue damage under multiaxial loading," International Journal of Fatigue, vol. 30, no. 9, pp. 1646-1658, 2008.

[2] A. Karolczuk and E. Macha, "Selection of the critical plane orientation in two-parameter multiaxial fatigue failure criterion under combined bending and torsion," Engineering Fracture Mechanics, vol. 75, no. 3-4, pp. 389-403, 2008. 
[3] D. Tchankov, M. Sakane, T. Itoh, and N. Hamada, "Crack opening displacement approach to assess multiaxial low cycle fatigue," International Journal of Fatigue, vol. 30, no. 3, pp. 417425, 2008.

[4] Y. S. Garud, "Multiaxial fatigue: a survey of the state of the art," Journal of Testing and Evaluation, vol. 9, no. 3, pp. 165-178, 1981.

[5] M. A. Miner, "Cumulative damage in fatigue," Journal of Applied Mechanics, vol. 67, pp. A159-A164, 1945.

[6] S. DeGuang, Research of Multiaxial Fatigue Damage and Fatigue Life Prediction, vol. 122, Northeastern University, ShenYang, China, 1996.

[7] J. Papuga, "A survey on evaluating the fatigue limit under multiaxial loading," International Journal of Fatigue, vol. 33, no. 2, pp. 153-165, 2011.

[8] F. Morel, "Critical plane approach for life prediction of high cycle fatigue under multiaxial variable amplitude loading," International Journal of Fatigue, vol. 22, no. 2, pp. 101-119, 2000.

[9] I. V. Papadopoulos, "Long life fatigue under multiaxial loading," International Journal of Fatigue, vol. 23, no. 10, pp. 839-849, 2001.

[10] S. Murakami, "Notion of continuum damage mechanics and its application to anisotropic creep damage theory," Journal of Engineering Materials and Technology, vol. 105, no. 2, pp. 99$105,1983$.

[11] D. Krajcinovic, Damage Mechanics, North-Holland, Elsevier, Amsterdam, The Netherlands, 1996.

[12] P. M. Mariano and G. Augusti, "Some axioms and theorems in damage mechanics and fatigue of materials," International Journal of Solids and Structures, vol. 34, no. 25, pp. 3337-3350, 1997.

[13] Y.-C. Xiao, S. Li, and Z. Gao, "A continuum damage mechanics model for high cycle fatigue," International Journal of Fatigue, vol. 20, no. 7, pp. 503-508, 1998.

[14] S. Dhar, P. M. Dixit, and R. Sethuraman, "A continuum damage mechanics model for ductile fracture," International Journal of Pressure Vessels and Piping, vol. 77, no. 6, pp. 335-344, 2000.

[15] R. K. Abu Al-Rub and G. Z. Voyiadjis, "On the coupling of anisotropic damage and plasticity models for ductile materials," International Journal of Solids and Structures, vol. 40, no. 11, pp. 2611-2643, 2003.

[16] J. D. Clayton, "Continuum multiscale modeling of finite deformation plasticity and anisotropic damage in polycrystals," Theoretical and Applied Fracture Mechanics, vol. 45, no. 3, pp. 163-185, 2006.

[17] A. Karolczuk and E. Macha, "A review of critical plane orientations in multiaxial fatigue failure criteria of metallic materials," International Journal of Fracture, vol. 134, no. 3-4, pp. 267-304, 2005.

[18] Y. Liu and S. Mahadevan, "Multiaxial high-cycle fatigue criterion and life prediction for metals," International Journal of Fatigue, vol. 27, no. 7, pp. 790-800, 2005.

[19] M. Zhang, Q. Meng, W. Hu, S. Shi, M. Hu, and X. Zhang, "Damage mechanics method for fatigue life prediction of PitchChange-Link," International Journal of Fatigue, vol. 32, no. 10, pp. 1683-1688, 2010.

[20] D. Krajcinovic, "Damage mechanics: accomplishments, trends and needs," International Journal of Solids and Structures, vol. 37, no. 1-2, pp. 267-277, 1999.

[21] H. Y. Zhou Taiquan, "Fatigue life evaluation for steel welded components using damage mechanics and finite element method," Industrial Construction, vol. 35, pp. 843-848, 2005 (Chinese).
[22] H. D. Espinosa, P. D. Zavattieri, and S. K. Dwivedi, "A finite deformation continuum/discrete model for the description of fragmentation and damage in brittle materials," Journal of the Mechanics and Physics of Solids, vol. 46, no. 10, pp. 1909-1942, 1998.

[23] R. Desmorat, A. Kane, M. Seyedi, and J. P. Sermage, "Two scale damage model and related numerical issues for thermomechanical High Cycle Fatigue," European Journal of Mechanics, A/Solids, vol. 26, no. 6, pp. 909-935, 2007.

[24] C. L. Chow and J. Wang, "An anisotropic theory of elasticity for continuum damage mechanics," International Journal of Fracture, vol. 33, no. 1, pp. 3-16, 1987.

[25] C. L. Chow and J. Wang, "An anisotropic theory of continuum damage mechanics for ductile fracture," Engineering Fracture Mechanics, vol. 27, no. 5, pp. 547-558, 1987.

[26] S. Wei, P. Li-Hua, Y. Yun-Guo, S. Zeng, and T. Xian-Dong, "Elastic damage and energy dissipation in anisotropic solid material," Engineering Fracture Mechanics, vol. 33, no. 2, pp. 273-281, 1989.

[27] F. Gao, Q. Wang, and X. Zhang, "Anisotropic damage mechanics with application to bolt fatigue life estimation," Chinese Journal of Applied Mechanics, vol. 24, no. 1, pp. 128-132, 2007 (Chinese).

[28] T. Hoshide and D. F. Socie, "Mechanics of mixed mode small fatigue crack growth," Engineering Fracture Mechanics, vol. 26, no. 6, pp. 841-850, 1987.

[29] H. Yu and X. Wu, The Material Databook of Aeroengine Design, Aviation Industry Press, 2010. 

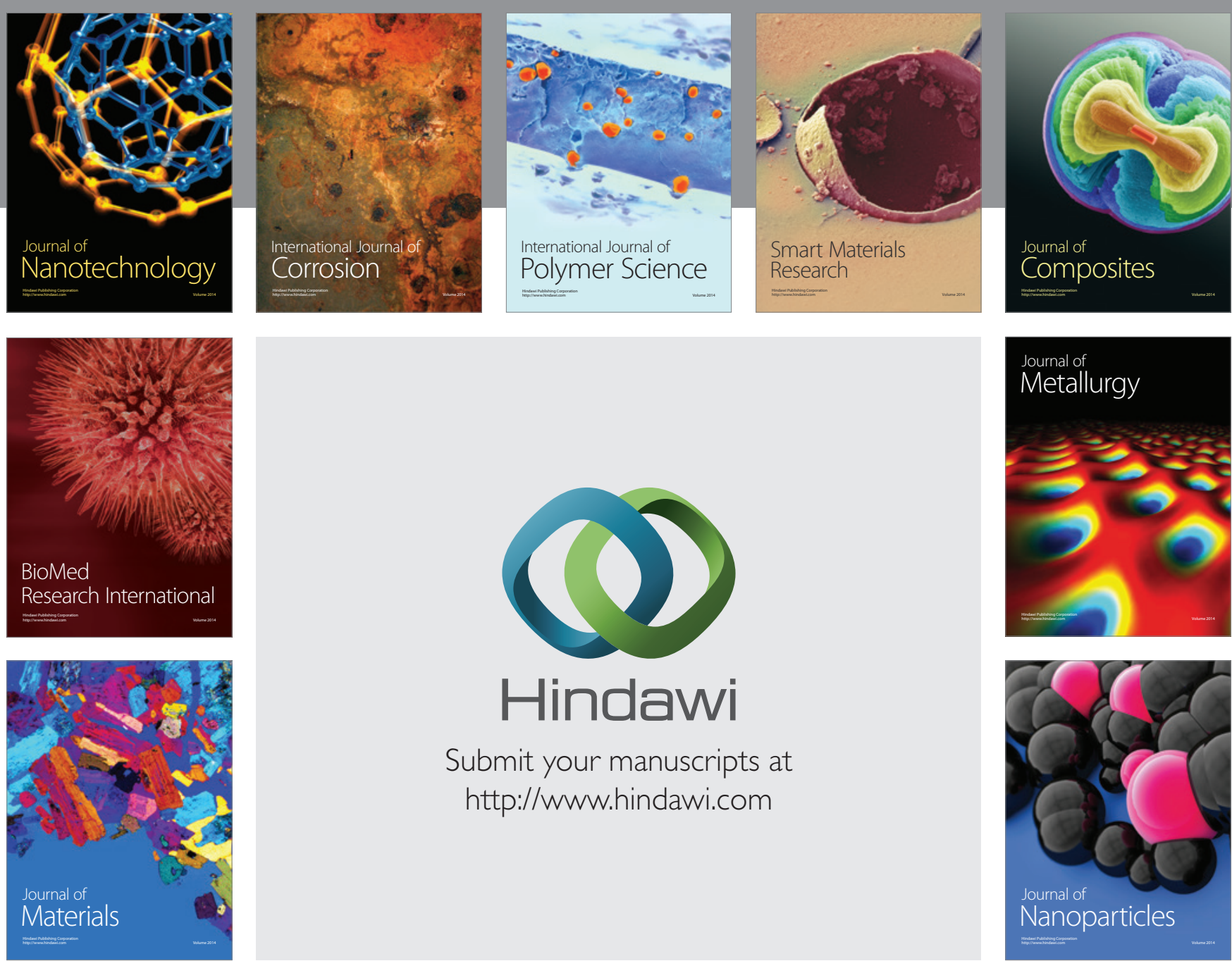

\section{Hindawi}

Submit your manuscripts at

http://www.hindawi.com

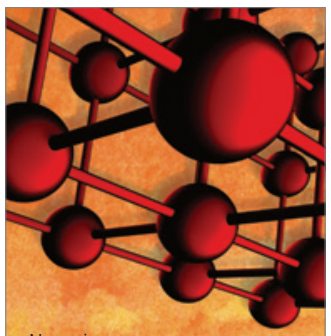

Materials Science and Engineering
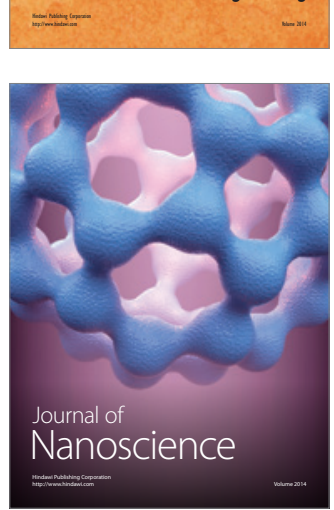
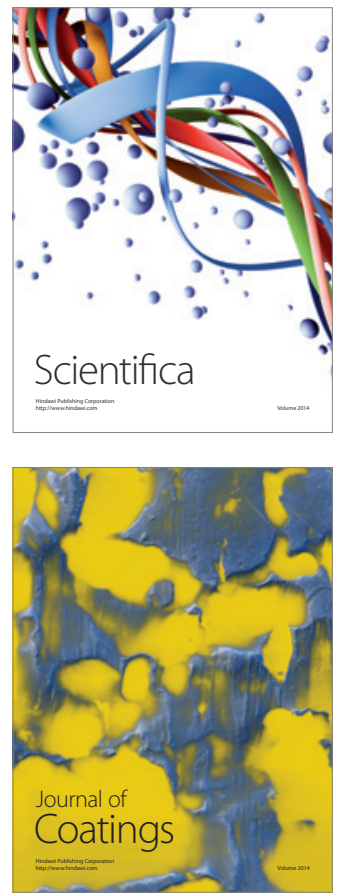
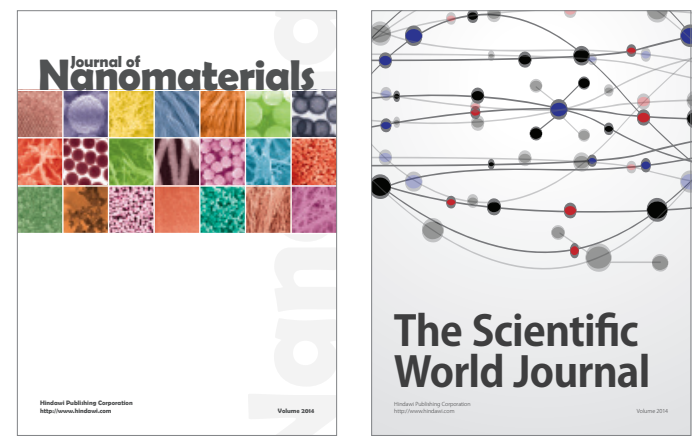

The Scientific World Journal
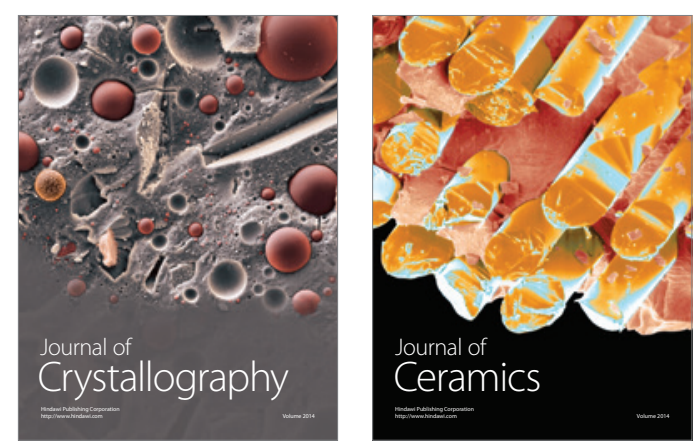
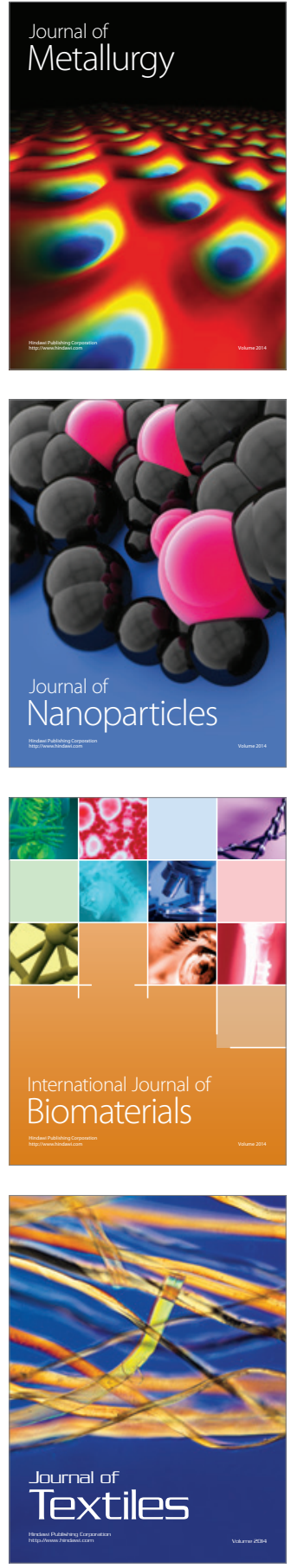